\title{
INSTANTONS AND AFFINE ALGEBRAS I: THE HILBERT SCHEME AND VERTEX OPERATORS
}

\section{Grojnowski}

A вstract. This is the first in a series of papers which describe the action of an affine Lie algebra with central charge $n$ on the moduli space of $U(n)$ instantons on a four manifold $X$. This generalises work of Nakajima, who considered the case when $X$ is an ALE space. In particular, this should describe the combinatorial complexity of the moduli space as being precisely that of representation theory, and thus will lead to a description of the Betti numbers of moduli space as dimensions of weight spaces.

This Lie algebra acts on the space of conformal blocks (i.e., the cohomology of a determinant line bundle on the moduli space [LMNS]) generalising the "insertion" and "deletion" operations of conformal field theory, and indeed on any cohomology theory.

In the particular case of $U(1)$-instantons, which is essentially the subject of this present paper, the construction produces the basic representation after Frenkel-Kac. Then the well known quadratic nature of $c h_{2}$,

$$
c h_{2}=\frac{1}{2} c_{1} \cdot c_{1}-c_{2}
$$

becomes precisely the formula for the eigenvalue of the degree operator, i.e. the well known quadratic behaviour of affine Lie algebras.

\section{Introduction}

This is the first in a series of papers devoted to describing the action of an affine Lie algebra on the moduli space of instantons on an algebraic surface $X$. This paper, which is only an announcement, is concerned with the "boundary" of moduli space; the subsequent papers will describe the action on the interior.

We describe the idea briefly. Let $X$ be an algebraic surface, $\mathcal{M}$ the moduli space of $U(c)$-instantons on $X$ (see below for precise definitions). $\mathcal{M}$ is not connected; it decomposes into $\mathcal{M}=\amalg \mathcal{M}_{c_{1}, c h_{2}}$, where $\mathcal{M}_{c_{1}, c h_{2}}$ denotes those instantons with fixed first Chern class equal to $c_{1} \in H^{2}(X, \mathbf{Z})$ and second Chern character equal to $\operatorname{ch}_{2} \in \mathbf{Q}=H^{4}(X, \mathbf{Q})$.

Received July 6, 1995. 
Let $\Sigma \subseteq X$ be an algebraic curve. Associated to $\Sigma$ we have various correspondences $\mathcal{M} \leftarrow \mathcal{P}_{\Sigma} \rightarrow \mathcal{M}$. Such a correspondence induces maps $H(\mathcal{M}) \rightarrow H(\mathcal{M})$ for any cohomology theory $H$. To describe these individual maps is very complicated. However, these maps satisfy very simple commutation relations, namely those defining a Lie algebra. Hence, shifting our point of view slightly, we see that $H(\mathcal{M})$ is a representation of this Lie algebra. This explains the complexity of the individual maps - they are the same as the (known) complexity of describing the action of a Lie algebra on the individual weight spaces of a representation.

What then needs to be described is the correspondences, the Lie algebra they generate, and which representations occur. The Lie algebras are affine Lie algebras, defined by the lattice $H^{2}(X, \mathbf{Z})$, or various sublattices such as the lattices of algebraic cycles (Neron-Severi group). These lattices become the "finite part" of the weight lattice, with the degree operator taking value in $H^{4}(X, \mathbf{Z})$. (The presence of affine Lie algebras, whose characters are known to be modular forms, is reassuringly consistent with the remarkable work [VW], which predicts this behaviour because of $S$-duality).

The description of the representation will have to wait for a future paper. I hope that it is irreducible, and determined by the Kähler cone and Kronheimer-Mrowka basic classes (choice of "positive roots" and "highest weight"). However, the most important invariant of a representation is its central charge, and in this paper we show that this charge is precisely the rank of the instanton (i.e. $U(n)$-instantons give rise to level $n$ representations).

The most basic example of a correspondence is the "elementary modifications"; i.e. given a divisor $i: \Sigma \hookrightarrow X$ we modify a vector bundle along $\Sigma$, that is consider the correspondence

$$
\mathcal{P}_{\Sigma}^{n}=\left\{0 \rightarrow \mathcal{A}_{1} \rightarrow \mathcal{A}_{2} \rightarrow i_{*} \mathcal{E} \rightarrow 0 \mid \mathcal{A}_{r} \in \mathcal{M}, \mathcal{E} \in \operatorname{Pic}^{n} \Sigma\right\}
$$

where $\operatorname{Pic}^{n} \Sigma$ is the moduli space of holomorphic line bundles on $\Sigma$ with degree $n$.

In the case where $X$ is a curve, divisors are points, and the analogous correspondences are precisely the geometric Hecke operators of Drinfeld. Points however cannot interact, whereas curves on surfaces most definitely do - their interaction being precisely described by the lattice $H^{2}(X, \mathbf{Z})$. It was the basic observation of Nakajima, in the case of an ALE space $X$, that the interactions of the correspondences are described by the Serre relations.

Variants of these correspondences $\mathcal{P}_{\Sigma}^{n}$ are the subject of the sequel to this paper.

This paper is concerned with a simpler correspondence, which removes a point from an instanton to produce a new one. As a vector bundle modified 
along a point becomes only a torsion free sheaf, and not a vector bundle, one should think of this correspondence as acting along the "boundary" of the moduli space. We find that the algebra generated by this correspondence is essentially an affine Heisenberg Lie algebra $(\S 3,5)$.

Obviously, these correspondences are well known in the literature. For example, the elementary modifications along a divisor appear in [MO], where they stratify $\mathcal{M}$ by what strongly looks like paths to the highest weight vector of a representation (i.e. by the "crystal" basis), and notably in $[\mathrm{KM}]$ where they are used to impose enough relations on the Donaldson polynomials to determine them in terms of certain basic classes.

Even our simple correspondence of inserting a point is a common technical tool; see for example [GL]. Thus our main contribution is to insist that one should study the algebra of these correspondences, and that this is easy.

Finally, these correspondences act on any cohomology theory. In the most intersting case, the cohomology of $\mathcal{M}$ with coefficents in a determinantal line bundle, one produces the action of an affine algebra on the space of conformal blocks ${ }^{1}$. This space, for which there is now a dimension formula [LMNS] generalising that of Verlinde for curves, has staggering implications for representation theory.

Acknowledgements: It should be clear that this paper is inspired by Nakajima's fantastic work [Na]. It is a pleasure to acknowledge helpful conversations with A. Beilinson, R. Dijkgraaf, L. Fastenberg, I. Frenkel, D. Gieseker, P. Kronheimer, Jun Li, A. Losev, G. Moore, N. Nekrasov and S. Shatashvili; and support both intellectual and moral from I. Frenkel and G. Moore, without which this work would not have occurred. Portions of these results were announced at talks at UCLA in November 1994 and UNC-Chapel Hill in April 1995. Nakajima independantly in the paper alg-geom/9507012 has proved a result similar to theorem 2 below.

\section{Algebraic preliminaries}

Let $V$ be a complex vector space, $t^{-1} V\left[t^{-1}\right]=V \otimes_{\mathbf{C}} t^{-1} \mathbf{C}\left[t^{-1}\right]$ the associated space of loops at $V$ which vanish at $\infty$. We make this a graded vector space by setting $\operatorname{deg}\left(v \otimes t^{n}\right)=-n$. We write $v_{n}$ for $v \otimes t^{n}$.

Let $S=S\left(t^{-1} V\left[t^{-1}\right]\right)=\oplus S_{n}$ be the graded polynomial algebra in infinitely many variables. We make this a Hopf algebra by defining $\Delta v_{n}=$ $v_{n} \otimes 1+1 \otimes v_{n}$, for $v \in V$. Then $S$ is a free commutative and cocommutative Hopf algebra. Conversely, given such a Hopf algebra $S$, we can reconstruct $V \otimes t^{-n}$ uniquely as the space of primitive elements of degree $n$.

\footnotetext{
${ }^{1}$ This paragraph is the consequence of conversations with Greg Moore; see [LMNS].
} 
Now suppose we are given a graded symmetric bilinear form $($,$) on S$ such that multiplication and comultiplication are adjoint, i.e. such that $\left(S_{n}, S_{m}\right)=0$ if $n \neq m$, and $(x y, z)=(x \otimes y, \Delta z)$ (where $S \otimes S$ inherits a bilinear form by $(x \otimes y, a \otimes b)=(x, a)(y, b))$. Such a form is completely specified by its values on the primitive elements, i.e. by the values $\left(\alpha_{n}^{i}, \alpha_{n}^{j}\right)$, where $\alpha^{i}$ runs through a basis of $V$.

Given a non-degenerate such form, we can define the action of $t V[t]=$ $V \otimes t \mathbf{C}[t]$ on $S$, by defining $v_{n}$ to be the adjoint of $v_{-n}$ for $n>0$, i.e.

$$
\left(v_{n} x, y\right)=\left(x, v_{-n} y\right), \quad n>0 .
$$

As $\Delta$ is an algebra homomorphism, it follows that $v_{n}$ acts as a derivation on $S$,

$$
\begin{aligned}
& \left(v_{n}(x y), z\right)=\left(x y, v_{-n} z\right)=\left(x \otimes y, \Delta\left(v_{-n} z\right)\right)=\left(x \otimes y,\left(v_{-n} \otimes 1+1 \otimes v_{-n}\right) \Delta z\right) \\
& =\left(v_{n} x \otimes y+x \otimes v_{n} y, \Delta z\right)=\left(v_{n}(x) y+x v_{n}(y), z\right)
\end{aligned}
$$

and hence that the Heisenberg Lie algebra $\left(\oplus_{n \neq 0} V \otimes t^{n}\right) \oplus \mathbf{C}$ acts on $S$, where $\left[v_{n}, w_{m}\right]=\delta_{n,-m} \cdot\left(v_{n}, w_{n}\right)$ if $n>0$.

Given $v \in V$, we define new elements $h_{n}^{v} \in S_{n}$ by

$$
H^{v}(t)=\sum_{n \geq 0} h_{n}^{v} t^{n}=\exp \left(\sum_{n \geq 1} v_{n} t^{n} / n\right) .
$$

Then if the elements $\alpha^{i}$ form a basis of $V$, it is well known that the elements $h_{n}^{\alpha^{i}}$ are algebraically independent and generate $S$. As $t^{-1} V\left[t^{-1}\right]$ consists of primitive elements, the $H^{v}(t)$ are 'group-like', i.e.

$$
\Delta h_{n}^{v}=\sum_{0 \leq a \leq n} h_{a}^{v} \otimes h_{n-a}^{v}
$$

and the inner product is given by

$$
\sum_{n, m \geq 0}\left(h_{n}^{v}, h_{m}^{w}\right) t^{n} s^{m}=\exp \left(\sum_{n \geq 1}\left(v_{n}, w_{n}\right) / n \cdot(t s)^{n} / n\right) .
$$

This infinite family of Heisenberg Lie algebras just constructed is still rather flabby; however inside this space of algebras (parametrised by maps from $\mathbf{Z}_{+}$to non-degenerate quadratic forms on $V$ ) there are certain remarkable families with much larger symmetries; namely the vertex algebras [B,FLM] and $q$-vertex algebras [FJ]. 
We suppose given a lattice $L$ with non-degenerate symmetric even bilinear form, i.e. $(\alpha, \alpha) \in 2 \mathbf{Z}$ for $\alpha \in L$, and put $V=L \otimes_{\mathbf{Z}} \mathbf{C}$. Define $($,$) on$ $t^{-1} V\left[t^{-1}\right]$ by

$$
\left(v_{n}, w_{m}\right)=(-1)^{n+1} n(v, w) \delta_{n, m} .
$$

With this inner product,

$$
\sum_{n, m \geq 0}\left(h_{n}^{v}, h_{m}^{w}\right) t^{n} s^{m}=(1+t s)^{(v, w)} .
$$

We call $S$ the Fock space modeled on the lattice $L$.

We also suppose given a two-cocycle $\epsilon: L \times L \rightarrow \mathbf{Z} / 2 \mathbf{Z}$, and define the group algebra of $L$ twisted by $\epsilon, \mathbf{C}\{L\}$, as in [FK,FLM]. Define $\mathcal{F}=$ $S \otimes_{\mathbf{C}} \mathbf{C}\{L\}$, a $\mathbf{Z}_{+} \times L$ graded vector space which carries an action of both the Heisenberg Lie algebra $\left(\oplus_{n \neq 0} V \otimes t^{n}\right) \oplus \mathbf{C}$ and $\mathbf{C}\{L\}$. Let $V$ act on $\mathbf{C}\{L\}$ by $v \cdot e^{\lambda}=(v, \lambda) e^{\lambda}$, where $\lambda \in L, e^{\lambda}$ denotes the corresponding element of the group algebra, and $v \in V$.

Then in these circumstances we have the well known result that a far larger algebra acts on $\mathcal{F}$, namely

Theorem [FLM,B]. $\mathcal{F}$ is a vertex algebra, and if $L$ is positive definite, a vertex operator algebra ${ }^{2}$

For example, in the particular case where $L$ is positive definite and spanned by the roots $\Delta=\{\alpha \in L \mid(\alpha, \alpha)=2\}$, then the space of vectors of conformal weight 1 is isomorphic to the simple Lie algebra $\mathfrak{g}$ with roots $\Delta$, and $\mathcal{F}$ is the basic representation of $\widehat{\mathfrak{g}}[\mathrm{FK}]$.

If $L$ is of arbitrary signature things become much more complicated.

At present, there is apparantly no general definition for a quantum vertex algebra. But if $L$ is positive definite and spanned by the roots, then we can follow $[\mathrm{FJ}]$ and define, for $c \in \mathbf{N}$

$$
\left(\alpha_{n}^{i}, \alpha_{m}^{j}\right)=n \delta_{n m}\left[n c\left(\alpha^{i}, \alpha^{j}\right)\right] /[n]
$$

where $[n]=\frac{q^{n}-q^{-n}}{q-q^{-1}}$, and $\alpha^{1}, \ldots, \alpha^{l}$ are a basis of simple roots. (There is an additional choice here, that of a positive cone in $V$ ). When $c=1$ this gives $\mathcal{F}$ the structure of the basic representation for $U_{q} \widehat{\mathfrak{g}}[\mathrm{FJ}]$.

This $q$ arises in our situation when there is some "weight" structure on the cohomology theory, for example a $\mathbf{C}^{*}$-action on $X$. This does occur for ALE spaces (see [N, Gr1]) but we will stick to $q=1$ for the present paper.

\footnotetext{
${ }^{2}$ If $L$ is $\mathbf{Z} / 2$-graded we may also make all these definitions, as long as we work in the $\mathbf{Z} / 2$-graded category. So $S$ is the free super-commutative algebra on $t^{-1} V\left[t^{-1}\right]$, i.e. a tensor product of an exterior algebra and a polynomial algebra, $\mathcal{F}$ is a super-vertex algebra, etc.
} 


\section{Motivic algebras}

We write this section with the minimal generality needed for this paper. Suppose $X$ and $Y$ are two smooth proper varieties, and $Z \subseteq X \times Y$ is a subvariety, i.e. a correspondence between $X$ and $Y$. We write this $X \leftarrow$ $Z \rightarrow Y$, and $\pi_{X}, \pi_{Y}$ for the two projections from $X \times Y$ to $X$ or $Y$.

Then if $H$ is any "reasonable" cohomology theory we obtain honest maps

$$
R: H(X) \rightarrow H(Y), \quad \bar{R}: H(Y) \rightarrow H(X)
$$

which are adjoint with respect to the natural inner product on $H(X)$ and $H(Y) ;(R a, b)=(a, \bar{R} b)$. Here we define $R(a)=\left(\pi_{Y}\right)_{*}\left(\pi_{X}^{*} a \cdot[Z]\right), \bar{R}(b)=$ $\left(\pi_{X}\right)_{*}\left([Z] \cdot \pi_{Y}^{*} b\right)$, and $\left(a, a^{\prime}\right)=\int_{*} a \cdot a^{\prime}$, for $a, a^{\prime} \in H(X), b \in H(Y)$ and where for any space $X, \int: X \rightarrow p t$ denotes the projection to a point, and $[Z]$ denotes the class of $Z$ in $H(X \times Y)$.

We mention some reasonable cohomology theories:

i) Usual homology or cohomology $H^{*}$; topological $K$-theory,..., cobordism, all with (say) complex coefficients.

ii) Write $\mathcal{F}(X)$ for the ring of constructible functions from $X$ to $\mathbf{C}$. If $\pi: X \rightarrow Y$ is a map, $f \in \mathcal{F}(Y), g \in \mathcal{F}(X)$, define $\left(\pi^{*} f\right)(x)=f(\pi(x))$ and $\left(\pi_{*} g\right)(y)=\sum_{a \in \mathbf{C}} a \chi\left(\pi^{-1}(y) \cap g^{-1}(a)\right)$, where $\chi$ denotes the Euler characteristic of cohomology with compact supports, and define $[Z]$, for $Z \subseteq X$ a subvariety, as the characteristic function of $Z:[Z](x)=1$ if $x \in Z$, and $[Z](x)=0$ otherwise.

iii) If $\operatorname{dim} Z=(\operatorname{dim} X+\operatorname{dim} Y) / 2$, then

$$
H^{\frac{1}{2} \operatorname{dim} X}(X) \underset{\bar{R}}{\stackrel{R}{\rightleftarrows}} H^{\frac{1}{2} \operatorname{dim} Y}(Y)
$$

where $H^{*}$ is the usual cohomology. If $X$ is compact Kähler (respectively complex algebraic or symplectic) we can consider the subspace of $H^{\frac{1}{2} \operatorname{dim} X}(X)$ spanned by the $(p, p)$-classes (respectively, algebraic or Lagrangian cycles). Denote any of these subspaces $H^{\Lambda}(X)$. As long as $Z$ is algebraic or Lagrangian as appropriate, the topological $R, \bar{R}$ preserve these subspaces and the theories $H^{\Lambda}(X)$ are "reasonable" cohomology theories.

As part of our definition of reasonable we require that the Kunneth map $H(X) \otimes H(Y) \rightarrow H(X \times Y), a \otimes b \mapsto \pi_{X}^{*} a \cdot \pi_{Y}^{*} b$ is an isomorphism. Here, if $H$ is $\mathbf{Z} / 2$-graded then we take $\otimes$ in the $\mathbf{Z} / 2$-graded sense also (as in example (i)).

One can continue this list of theories as one pleases [JKS]. The above theories all take values in vector spaces, as our goal is to produce representations of algebras, but if the correspondences act non-trivially on the 
entire motives of $X$ and $Y$ one should also consider functors which do not factor through cycles homologically equivalent to zero.

An example not on this list, but which I hope to return to, is homology of $X$ with coefficients in a given sheaf. In our case below, the sheaf should be taken to be a determinant line bundle on the moduli space of torsion free sheaves, so that its cohomology is the space of conformal blocks. Then the geometric Hecke operators (quantum group symmetries) we produce act on the representation theory of the double loop groups in some as yet unknown way.

\section{Hilbert schemes}

We recall some well known facts about the Hilbert scheme. Let $X$ be a smooth algebraic surface, $S^{n} X=X^{n} / S_{n}$ the $n$ 'th symmetric power of $X$. For $n>1, S^{n} X$ is singular. Write $\widetilde{S^{n} X}$ for the Hilbert scheme of $X$, i.e. for the variety parameterising closed zero dimensional subschemes of $X$ of length $n$, and let $\pi: \widetilde{S^{n} X} \rightarrow S^{n} X$ be the canonical morphism sending a subscheme to its support. Then $\widetilde{S^{n} X}$ actually exists as a separated variety; it is smooth of dimension $2 n$ [Gro,Fo], $\pi$ is proper and produces a desingularisation of $S^{n} X$.

If $X$ is symplectic then so is $\widetilde{S^{n} X}$, if $X$ is hyper-Kähler than $\widetilde{S^{n} X}$ is the hyper-Kähler resolution of the stack (orbifold) $\left[S^{n} X\right]$. We phrase everything below in terms of the variety $\widetilde{S^{n} X}$, but it is often much better to work directly with the smooth stack $\left[S^{n} X\right]$.

If $x \mapsto n x$ denotes the diagonal map $X \rightarrow X^{n} \rightarrow S^{n} X$, then $\pi^{-1}(n x)$ is irreducible, and of dimension $n-1$ [Br]. This fact was used in [GS] to compute the Hodge numbers of $\widetilde{S^{n} X}$ in terms of those of $X$; a description of the Euler numbers which is in the spirit of this paper can be found in [VW].

Let $\mathcal{P}_{n}$ denote the set of partitions of $n$. If $\alpha=\left(1^{\alpha_{1}} 2^{\alpha_{2}} \ldots\right) \in \mathcal{P}_{n}$, so $\sum_{i} i \alpha_{i}=n$, write $\ell(\alpha)=\sum_{i} \alpha_{i}$. We have an obvious stratification of $S^{n} X$ by $\mathcal{P}_{n}$; the strata $S_{\alpha}^{n} X$ has complex dimension $2 \ell(\alpha)$. Write $\widetilde{S_{\alpha}^{n} X}$ for $\pi^{-1}\left(S_{\alpha}^{n} X\right)$. Then $\mathcal{A} \in \widetilde{S_{\alpha}^{n} X}$ if $\mathcal{A}$ is isomorphic to a direct sum $\oplus \mathcal{A}_{i, r}$, where $1 \leq r \leq \alpha_{i}$, each $\mathcal{A}_{i, r} \in \widetilde{S^{i} X}$ has support a single point with multiplicity $i, \pi\left(\mathcal{A}_{i, r}\right)=i \gamma_{i, r}$, and the points $\gamma_{i, r}$ are distinct. We have $\operatorname{dim} \widetilde{S_{\alpha}^{n} X}=n+\ell(\alpha)$.

The open strata $S_{\left(1^{n}\right)}^{n}, \widetilde{S_{\left(1^{n}\right)}^{n} X}$ we also denote $\left(S^{n} X\right)^{0}, \widetilde{\left(\widetilde{S^{n} X}\right)^{0}} ; \pi$ restricted to $\left(\widetilde{S^{n} X}\right)^{0}$ is an isomorphism. 
Define

$$
\Lambda^{0}=\Lambda_{a b}^{0}=\left\{\left(\mathcal{A}_{1}, \mathcal{A}_{2}, \mathcal{A}_{3}\right) \in \widetilde{S^{a} X} \times \widetilde{S^{a+b} X} \times \widetilde{S^{b} X}\right.
$$

$\mathcal{A}_{2} \in\left(\widetilde{S^{a+b} X}\right)^{0}$, and there is an exact sequence $\left.0 \rightarrow \mathcal{A}_{1} \rightarrow \mathcal{A}_{2} \rightarrow \mathcal{A}_{3} \rightarrow 0\right\}$

and define $\Lambda$ to be the closure of $\Lambda^{0}$ in $\widetilde{S^{a} X} \times \widetilde{S^{a+b} X} \times \widetilde{S^{b} X}$. Observe that

i) If $\left(\mathcal{A}_{1}, \mathcal{A}_{2}, \mathcal{A}_{3}\right) \in \Lambda^{0}$, then $\mathcal{A}_{1}, \mathcal{A}_{2}$ and $\mathcal{A}_{3}$ are all in the open stratum of their Hilbert schemes.

ii) We have $\left(\mathcal{A}_{1}, \mathcal{A}_{2}, \mathcal{A}_{3}\right) \in \Lambda_{a b}$ if and only if $\left(\mathcal{A}_{3}, \mathcal{A}_{2}, \mathcal{A}_{1}\right) \in \Lambda_{b a}$.

iii) Writing $+: S^{a} X \times S^{b} X \rightarrow S^{a+b} X$ for the obvious morphism, we have $\pi\left(\mathcal{A}_{2}\right)=\pi\left(\mathcal{A}_{1}\right)+\pi\left(\mathcal{A}_{3}\right)$, if $\left(\mathcal{A}_{1}, \mathcal{A}_{2}, \mathcal{A}_{3}\right) \in \Lambda$. In fact $\Lambda$ is just the correspondence of varieties produced by the "obvious" correspondence of stacks

$$
\Lambda^{\text {stack }}=\left\{\left(\mathcal{A}_{1}, \mathcal{A}_{2}, \mathcal{A}_{3}\right) \mid \mathcal{A}_{2}=\mathcal{A}_{1}+\mathcal{A}_{3}\right\} .
$$

This is the basic motivic object, from which everything else follows.

iv) The dimension of $\Lambda$ is $2(a+b)$, i.e. half the dimension of the ambient space. In fact,

Lemma 1. If $X$ is symplectic, then $\Lambda$ is Lagrangian (where we change the sign of the symplectic form on $\widetilde{S^{a+b} X}$ in $\widetilde{S^{a} X} \times \widetilde{S^{a+b} X} \times \widetilde{S^{b} X}$ as is usual).

Now, let $H$ be a reasonable cohomology theory as in $\S 2$. Write

$$
S=\oplus_{n \geq 0} H\left(\widetilde{S^{n} X}\right)
$$

Define, for $x \in \widetilde{S^{a} X}, y \in \widetilde{S^{b} X}$, the product of $x$ and $y$,

$$
x y=\left(\pi_{a+b}\right)_{*}\left(\left(\pi_{a}, \pi_{b}\right)^{*}(x \otimes y) \cdot[\Lambda]\right),
$$

and for $z \in \widetilde{S^{a+b} X}$, define

$$
\Delta_{a b} z=\left(\pi_{a}, \pi_{b}\right)_{*}\left([\Lambda] \cdot \pi_{a+b}^{*} z\right) \in H\left(\widetilde{S^{a} X}\right) \otimes H\left(\widetilde{S^{b} X}\right)
$$

and $\Delta=\sum_{a+b=n} \Delta_{a b}$. Also define a non-degenerate inner product $($,$) :$ $H\left(\widetilde{S^{n} X}\right) \times H\left(\widetilde{S^{m} X}\right) \rightarrow H(p t)$ by $(x, y)=\delta_{n m} \int_{*} x \cdot y$.

Theorem 2. Equipped with this multiplication and comultiplication, $S$ is a commutative and cocommutative Hopf algebra. In other words, multplication and comultipication are associative, adjoint with respect to the inner 
product, and (graded) commutative (here, if $H$ is $\mathbf{Z} / 2$-graded, so is each $\left.S_{n}\right) . \Delta$ is an algebra homomorphism.

The only statement that requires proof is that $\Delta$ is an algebra homomorphism; the rest comes free with the formalism. This is easiest proved by using the stacks $\left[S^{n} X\right]$ and the obvious correspondendence between them; then if the cohomology theory is the "orbifold cohomology" (which here is essentially $K$-theory of $X^{n}$, equivariant with respect to the symmetric group) this induces our correspondence in the homology of $\widetilde{S^{n} X}$. In fact, in this form the theorem makes sense for any variety $X$ (of any dimension), and the orbifold cohomology ${ }^{3}$; the remarkable thing about surfaces is the additional intepretation in terms of honest seperated smooth varieties.

We would now like to describe generators for $S$, and identify $S$ with a Fock space as in $\S 1$.

Let $\Sigma \subseteq X$ be a curve, $S^{n} \Sigma$ its $n^{\prime}$ th symmetric power. If $\Sigma$ is algebraic, we can canonically identify $S^{n} \Sigma$ with the Hilbert scheme of zero dimensional subschemes of $\Sigma$ of length $n$, so we can also regard $S^{n} \Sigma$ as contained in $\widetilde{S^{n} X}$. It is a smooth subvariety, and Lagrangian if $X$ is symplectic. Remarkably, the following generalisation appears to be new:

Write $\widetilde{\Sigma}$ for the subspace of points $x \in \widetilde{S^{n} X}$ such that $\pi(x) \in S^{n} \Sigma$, and $\widetilde{\Sigma}_{\lambda}^{0}$ for $\widetilde{\Sigma} \cap \widetilde{S_{\lambda}^{n} X}$, where $\lambda \in \mathcal{P}_{n}$, and $\widetilde{S_{\lambda}^{n} X}$ is the piece of stratification defined above. Explicitly, $\mathcal{A} \in \widetilde{S^{n} X}$ is in $\widetilde{\Sigma}_{\lambda}^{0}$ if $\lambda=\left(1^{\alpha_{1}} 2^{\alpha_{2}} \cdots\right)$, and $\mathcal{A}$ is isomorphic to a direct sum $\oplus \mathcal{A}_{i, r}$, where $1 \leq r \leq \alpha_{i}$, each $\mathcal{A}_{i, r} \in \widetilde{S^{i} X}$ has support a single point with multiplicity $i, \pi\left(\mathcal{A}_{i, r}\right)=i \gamma_{i, r}$, and the points $\gamma_{i, r}$ are distinct. Write $\widetilde{\Sigma}_{\lambda}$ for the closure of $\widetilde{\Sigma}_{\lambda}^{0}$ in $\widetilde{S^{n} X}$.

\section{Proposition 3.}

i) $\widetilde{\Sigma}$ has pure dimension $n, \widetilde{\Sigma}_{\lambda}^{0}$ has pure dimension $n$, and the $\widetilde{\Sigma}_{\lambda}, \lambda \in$ $\mathcal{P}_{n}$, are precisely the irreducible components of $\widetilde{\Sigma}$.

ii) If $X$ is symplectic, then $\widetilde{\Sigma}$ is a Lagrangian submanifold.

See $\S 6$ below for more remarks on $\widetilde{\Sigma}$.

Write $h_{n}^{\Sigma}$ for the class of $S^{n} \Sigma \hookrightarrow \widetilde{S^{n} X}$ inside $S$. (Note that as a consequence of the proposition, we can define this class even if $\Sigma$ is not algebraic, though we do not need to.) We adopt the convention that $h_{0}^{\Sigma}=1$, for all $\Sigma$.

\footnotetext{
${ }^{3}$ i.e. let $\mathcal{F}=\oplus_{n} K^{S_{n}}\left(X^{n}, \mathbf{C}\right)$, for $X$ any variety. Then the theorem is that $\mathcal{F}$ is a Fock space modeled on $H^{*}(X, \mathbf{C})$, with multiplication and comultiplication defined by the obvious correspondence. Like everything else stated here, the proof of this fact will appear in the longer version of this paper.
} 


\section{Proposition 4.}

i) The elements $h_{n}^{\Sigma}$ are group-like, i.e.

$$
\Delta h_{n}^{\Sigma}=\sum_{a+b=n} h_{a}^{\Sigma} \otimes h_{b}^{\Sigma}
$$

ii) Let $\Sigma, \Sigma^{\prime}$ be two curves in $X$. Then

$$
\sum_{n, m \geq 0}\left(h_{n}^{\Sigma}, h_{m}^{\Sigma^{\prime}}\right) t^{n} s^{m}=(1+t s)^{\left(\Sigma, \Sigma^{\prime}\right)}
$$

where $\left(\Sigma, \Sigma^{\prime}\right)$ denotes the inner product in $H(X)$, as usual.

Now, for $n \geq 0$ define $\Gamma^{X}=\Gamma_{n}^{X}=\widetilde{S_{(n)}^{n} X}$, a closed irreducible subvariety of $\widetilde{S^{n} X}$. So $\Gamma^{X}=\left\{\mathcal{A} \in \widetilde{S^{n} X} \mid \pi(\mathcal{A})=n x\right.$, for some $\left.x \in X\right\}=X \times{ }_{S^{n} X}$ $\widetilde{S^{n} X}$. Write $\pi: \Gamma^{X} \rightarrow X$, and define for any submanifold $Z \subseteq X, \Gamma^{Z}=$ $\Gamma_{n}^{Z} \subseteq \Gamma_{n}^{X}$ by $\Gamma^{Z}=\pi^{-1}(Z)$, i.e. so that the diagram

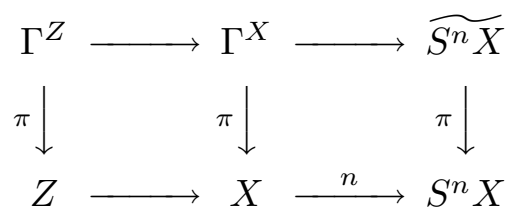

is Cartesian. In particular, if $\Sigma \subseteq X$ is an algebraic curve, $\Gamma^{\Sigma}=\widetilde{\Sigma}_{(n)}$ in the notation above. Also write $r_{n}^{Z}$ for the class of $\Gamma_{n}^{Z}$ in $H\left(\widetilde{S^{n} X}\right)$. We then have

Lemma 5. There exists a function $f: \mathbf{Z} \rightarrow \mathbf{Z}$, and polynomials in infinitely many variables $p_{a}^{n}\left(x_{1}, x_{2}, \ldots\right), q_{b}^{n}\left(x_{1}, x_{2}, \ldots\right)$ such that for any submanifolds $Z, Z^{\prime}$ of $X$,

$$
\begin{aligned}
& \text { i) }\left(r_{n}^{Z}, r_{n}^{Z^{\prime}}\right)=\left(Z, Z^{\prime}\right) f(n) \text {, and } \\
& \text { ii) } \Delta r_{n}^{Z}=\sum_{a+b=n} p_{a}^{n}\left(r_{1}^{Z}, r_{2}^{Z}, \ldots\right) \otimes q_{b}^{n}\left(r_{1}^{Z}, r_{2}^{Z}, \ldots\right)
\end{aligned}
$$

Now observe that for a fixed algebraic curve $\Sigma$, the algbera generated by the $r_{n}^{\Sigma}, n \in \mathbf{Z}_{+}$, is the same as the algebra generated by the $h_{n}^{\Sigma}, n \in \mathbf{Z}_{+}$. As a consequence of lemma 5 , we can write $h_{n}^{\Sigma}$ as a polynomial in the $r_{i}^{\Sigma}$ with "universal" coefficients; i.e. coefficients independent of $\Sigma$. This allows us to define $h_{n}^{Z}$, for any $Z \subseteq X$ by the same formulae. It then follows from lemma 5 and proposition 4 that the $h_{n}^{Z}$ are also group like, and satisfy the same inner product formulae as in proposition 4.

Take $H(X)=H^{*}(X)$ (usual cohomology), so $S=\oplus H^{*}\left(\widetilde{S^{n} X}\right)$, and let $Z_{i}, i=1, \ldots, l$ run through submanifolds of $X$ such that the classes $\left[Z_{i}\right]$ form a basis in $H^{*}(X, \mathbf{C})$. 
Proposition 6. The elements $r_{n}^{Z_{i}}$ freely generate $S$ as an algebra.

Now let $X$ be projective, so that the lattice $H^{*}(X, \mathbf{Z}) /$ torsion is a nondegenerate lattice with respect to the form $($,$) . We have thus proved$

Theorem 7. $S$ forms a Fock space modeled on the lattice $H^{*}(X, \mathbf{Z}) /$ torsion.

If $X$ is affine, one easily shows that if we take $H(X)=H_{\frac{1}{2} \operatorname{dim} X}(X, \mathbf{C})$ to be middle dimensional Borel-Moore homology, that the lattice $L=$ $H_{\frac{1}{2} \operatorname{dim} X}(X, \mathbf{Z})$ is non-degenerate and $S$ (for this cohomology theory) is a Fock space modeled on $L$. This is precisely the case that occurs for $X$ an ALE space.

For smooth projective $X$, let us agree to write $S^{\Lambda}=\oplus H^{\Lambda}\left(\widetilde{S^{n} X}\right)$, where $H^{\Lambda}$ as in $\S 2$ denotes either toplogical, $(n, n)$, holomorphic or Lagrangian middle dimensional cycles, and let us still write $S=\oplus H^{*}\left(\widetilde{S^{n} X}\right)$. Clearly $S^{\Lambda}$ is a Hopf subalgebra of $S$, and we can describe it explicitly using the above theorem.

For example, consider the Hodge decomposition of $H^{*}\left(\widetilde{S^{n} X}\right)$; write $S_{n}^{a b}=H^{a, b}\left(\widetilde{S^{n} X}\right)$. Then multiplication in $S$ preserves all this grading: $S_{n}^{a b} \cdot S_{m}^{c d} \subseteq S_{n+m}^{a+c, b+d}$. The generators $r_{n}^{Z} \in S_{n}$ have degree $(n-1, n-1)+$ $\operatorname{deg} Z$, i.e. if $[Z] \in H^{p, q}(X, \mathbf{C})$ then $r_{n}^{Z} \in S_{n}^{n+p-1, n+q-1}$. This gives another proof of [Got,3.1], independent of [GS].

Observe that $S^{\Lambda}$ is usually not a Fock space modeled on a lattice. For example, if the odd cohomology of $X$ vanishes, the generating function $\operatorname{dim} S_{n}^{n, n} z^{n}$ is the coefficent of $u^{0}$ in

$$
\prod_{n \geq 1}\left(\left(1-z^{n} u\right)\left(1-z^{n} u^{-1}\right)\right)^{-h^{2,0}}\left(1-z^{n}\right)^{-h^{1,1}}
$$

which is not the generating function of a Fock space.

\section{Vertex algebras and $U(1)$-instantons}

Let $X$ be a smooth projective surface. A torsion free sheaf $\mathcal{E}$ is a coherent sheaf of $\mathcal{O}_{X}$-modules which is torsion free as a $\mathcal{O}_{X}$-module. If $\mathcal{E}^{*}$ denotes the dual of $\mathcal{E}$, we have a canonical exact sequence $0 \rightarrow \mathcal{E} \rightarrow \mathcal{E}^{* *} \rightarrow \mathcal{Q} \rightarrow 0$, where $\mathcal{Q}$ is coherent of finite length, and $\mathcal{E}^{* *}$ is locally free. We say $\mathcal{E}$ has rank $c$ if $\mathcal{E}^{* *}$ does.

Let $H$ be a fixed very ample divisor on $X$, and $\mathcal{M}$ (resp. $\overline{\mathcal{M}}$ ) the space of $H$-stable rank $c$ torsion free sheaves (resp. $H$-semistable torsion free sheaves, modulo the usual equivalence relation $[\mathrm{Gi}])$. Then $\overline{\mathcal{M}}$ is a projective variety, and $\mathcal{M} \subseteq \overline{\mathcal{M}}$ an open subvariety [Gi]. Clearly $\overline{\mathcal{M}}=$ $\coprod_{c_{1}, k} \overline{\mathcal{M}}_{c_{1}, k}$, where $\overline{\mathcal{M}}_{c_{1}, k}$ consists of torsion free sheaves $\mathcal{E}$ with Chern 
classes $c_{1}(\mathcal{E})=c_{1}, \operatorname{ch}_{2}(\mathcal{E})=k$. Also, $c_{1}(\mathcal{E})$ lands in the lattice of algebraic cycles $c_{1}(\mathcal{E}) \in H^{\Lambda}(X, \mathbf{Z})$.

In the sequel we will be concerned with elementary modifications, and hence the interior of moduli space. For now, as we are only concerned with the boundary of moduli space, let us suppose $c=1$. Further, for simplicity, suppose $\pi_{1}(X)=0$. Then $H^{1}(X, \mathbf{Z})=H^{3}(X, \mathbf{Z})=0$, and $H^{2}(X, \mathbf{Z})$ is torsion free. Suppose also that $X$ is spin, so $H^{2}(X, \mathbf{Z})$ is even.

Now, in this case line bundles have no moduli, and so the space of torsion free sheaves is isomorphic to

$$
\oplus_{\lambda \in H^{\Lambda}(X, \mathbf{Z})}\left(\oplus_{n} \widetilde{S^{n} X}\right) \otimes e^{\lambda}
$$

Observe that the moduli of torsion free sheaves $\mathcal{E}$ such that $\mathcal{E}^{* *}$ is isomorphic to $\mathcal{L}$ is canonically isomorphic to those with $\mathcal{E}^{* *}$ isomorphic to $\mathcal{L}^{\prime}$; the map is just $\mathcal{E} \mapsto \mathcal{E} \otimes\left(\mathcal{L}^{\prime} \otimes \mathcal{L}^{-1}\right)$. This defines the action of the lattice $H^{\Lambda}(X, \mathbf{Z})$ on the moduli space of rank 1 torsion free sheaves. (If $X$ is not simply connected, we must replace this action by elementary modifications; this will be explained in the sequel).

Let us write $L=H^{*}(X, \mathbf{Z})$, and $\mathcal{M}^{\prime}=\left(\oplus_{n} \widetilde{S^{n} X}\right) \times L$,

$$
\mathcal{F}=\oplus H^{*}\left(\widetilde{S^{n} X}, \mathbf{C}\right) \otimes \mathbf{C}\{L\}=S \otimes \mathbf{C}\{L\}
$$

Then $\mathcal{M}^{\prime}$ is "almost" the moduli space of topological $U(1)$-instantons; i.e. the hyper-Kähler resolution of the ideal ASD-connections on toplogical $U(1)$-bundles. (It would be interesting to give a purely algebraic construction of this space). It differs from this by the term $H^{0}(X, \mathbf{Z})+H^{4}(X, \mathbf{Z})$, which I do not know how to intepret geometrically. Yet.

In any case, the results of $\S 3$ tell us

Theorem. $\mathcal{F}$ is a vertex algebra.

and that if $X$ is an ALE space, so $L=H^{2}(X, \mathbf{Z})$ is negative definite, that the analogously defined $\mathcal{F}$ (which is now precisely the homology of rank 1 torsion free sheaves) is just the basic representation of $\widehat{\mathfrak{g}}$, where $\widehat{\mathfrak{g}}$ is the affine Lie algebra associated to $L$.

Also, we remark that the theorem is true for an arbitrary compact Kähler 4-manifold, where we use the orbifold cohomology.

\section{Torsion free sheaves of rank $c$ produce central charge $c$}

Let $\mathcal{V}$ be a vector bundle of rank $c$ on $X$, and let $\mathcal{T}_{\mathcal{V}, n}$ consist of the stack of torsion free sheaves $\mathcal{E}$ such that $\mathcal{E}^{* *}$ is isomorphic to $\mathcal{V}$. Then if 
$\mathcal{V}$ is stable (resp. semistable), so is any $\mathcal{E} \in \mathcal{T}_{\mathcal{V}, n}$ (though not conversely), and in that case $\mathcal{T}_{\mathcal{V}, n}$ is a smooth separated variety.

Define $\mathcal{Q}_{\mathcal{V}}=\oplus_{n} H\left(\mathcal{T}_{\mathcal{V}, n}\right)$, where $H$ is any reasonable cohomology theory. One may handle $\mathcal{Q} \mathcal{V}$ as one handles $S$ in $\S 3$, by defining an action of $S$ on $\mathcal{Q}_{\mathcal{V}}, S \otimes \mathcal{Q}_{\mathcal{V}} \stackrel{m_{\mathcal{V}}}{\longrightarrow} \mathcal{Q}_{\mathcal{V}}$ induced by the correspondence

$$
\Lambda^{0}=\left\{\left(\mathcal{A}_{1}, \mathcal{A}_{2}, \mathcal{A}_{3}\right) \in \mathcal{T}_{\mathcal{V}, a} \times \mathcal{T}_{\mathcal{V}, a+b} \times \widetilde{S^{b} X} \mid\right.
$$

$\mathcal{A}_{2} \in \mathcal{T}_{\mathcal{V}, a+b}^{0}$, and there is an exact sequence $\left.0 \rightarrow \mathcal{A}_{1} \rightarrow \mathcal{A}_{2} \rightarrow \mathcal{A}_{3} \rightarrow 0\right\}$

and $\Lambda$ is the closure of $\Lambda^{0}$. Here, $\mathcal{T}_{\mathcal{V}, n}^{0}$ consists of those torsion free sheaves $\mathcal{E}$ which fit into an exact sequence $\mathcal{E} \rightarrow \mathcal{V} \rightarrow \mathcal{Q}$, where $\mathcal{Q} \in\left(\widetilde{S^{n} X}\right)^{0}$.

Then one may proceed exactly as in $\S 3$, and show

Theorem. $\mathcal{Q}_{\mathcal{V}}$ is a module for the Heisenberg Lie algebra $\left(\oplus_{n \neq 0} H^{*}(X, \mathbf{C}) \otimes t^{n}\right) \oplus \mathbf{C}$ with central charge $c$.

It is pleasant to calculate

$$
\left[h_{1}^{\Sigma}, h_{-1}^{\Sigma^{\prime}}\right]=c\left(\Sigma, \Sigma^{\prime}\right)
$$

directly. This follows directly from the easy fact that if $\mathcal{E}$ is any torsion free sheaf of generic rank $c$, and $\mathbf{C}_{0}$ denotes the skyscraper sheaf at a point $0 \in X$, then

Lemma. $\operatorname{dim} \operatorname{Ext}^{1}\left(\mathbf{C}_{0}, \mathcal{E}\right)+c=\operatorname{dim} \operatorname{Hom}\left(\mathcal{E}, \mathbf{C}_{0}\right)$.

In the particular case that $c=1$, this may be intepreted as (and in fact follows from) the fact that there is one way more to add a square to a partition than to remove a square. (As one may complete $X$ at 0 , to get $\mathbf{C}[[x, y]]$. This admits a $\mathbf{C}^{*}$-action, such that $x^{i} y^{j}$ have distinct weights for $i+j \leq n$. Then the fixpoints of this $\mathbf{C}^{*}$ action on the Hilbert scheme of length $n$ subschemes are just in 1-1 correspondence with partitions of $n$, and this punctual Hilbert scheme partitions into vector bundles over these isolated fixpoints. This makes the lemma obvious in this case, and gives yet another reason why the Hodge theory of $H^{*}\left(\widetilde{S^{n} X}\right)$ is so simple).

\section{Remarks on curves}

Suppose $\Sigma \subseteq X$ is an algebraic curve. Replace $X$ with the normal bundle to $\Sigma$ in $X$, so that $X$ admits a contracting $\mathbf{C}^{*}$ action with fixpoints $\Sigma$, that is if $x \in X, \lim _{t \rightarrow 0} t \cdot x$ exists and is in $\Sigma$. This $\mathbf{C}^{*}$ action induces one on $\widetilde{S^{n} X}$, and we define

$$
\mathcal{U}=\left\{\mathcal{A} \in \widetilde{S^{n} X} \mid \lim _{t \rightarrow 0} t \cdot x \in S^{n} \Sigma\right\}
$$

where $S^{n} \Sigma \hookrightarrow \widetilde{S^{n} X}$ as in $\S 3$. 


\section{Proposition.}

i) $\mathcal{U}$ is open in $\widetilde{S^{n} X}$, and $\mathcal{U}$ is a rank $n$ vector bundle on $S^{n} \Sigma$.

ii) Suppose $X=T^{*} \Sigma$. Then $\mathcal{U}$ canonically identifies with $T^{*}\left(S^{n} \Sigma\right)$. Under this identification, the Lagrangian subvariety $\widetilde{\Sigma} \cap \mathcal{U}$ identifies with Laumon's global nilpotent cone, a Lagrangian subvariety in $T^{*}\left(S^{n} \Sigma\right)$ [La].

Because of this proposition, a perverse sheaf on $S^{n} \Sigma$ with nilpotent characteristic variety (for example, conjecturally any automorphic sheaf) gives rise to a cycle in $H^{\Lambda}\left(\widetilde{S^{n} X}\right)$ via the characteristic cycle map.

Its also worth remarking that if $\Sigma$ is the affine line, $S^{n} \Sigma$ canonically identifies with the variety of regular conjugacy classes in $\mathfrak{g l}_{n}$ (via the characteristic polynomial). Thus $S^{n} \Sigma$, for $\Sigma$ a curve of genus $g$, which classically $^{4}$ one regards as a genus $g$ generalisation of regular conjugacy classes, is here generalised 'microlocally' to produce two dimensional analogues of conjugacy classes. (As the curve $\Sigma$ varies, this really does feel two dimensional).

Note that we do not want to consider the stack of coherent sheaves of length $n$ here (the analogue of the stack of all conjugacy classes in $\mathfrak{g l}_{n}$ ), as in our case the central charge would vary: $\mathfrak{Q u o t}_{V, r}$ has central charge the rank of $V$, where $V$ is a vector bundle on $X$.

\section{Remarks on Nakajima's quiver varieties and [Gr1]}

Let $\mathfrak{g}$ be a Kac-Moody Lie algebra, with symmetric Cartan matrix. In [L1], Lusztig defined a variety, the moduli space of representations of the quiver associated to $\mathfrak{g}$, and a Lagrangian subvariety $\Lambda$ such that the middle dimensional cycles on $\Lambda\left(H^{\Lambda}(\Lambda)\right.$ in the notation of $\left.\S 2\right)$ realises the universal Verma module for $\mathfrak{g}$.

In [Na] Nakajima constructed a modified quiver variety $\mathcal{M}_{\xi}(w)$, depending on a highest weight $w$ and a $\xi \in \mathfrak{h}$, where $\mathfrak{h}$ is the "real" Cartan subalgebra of $\mathfrak{g}$, with the following properties:

i) If $\xi$ is generic, then $H^{\Lambda}\left(\mathcal{M}_{\xi}(w)\right)$ realises the irreducible integrable highest weight module with highest weight $w$, and the Chevalley generators of $\mathfrak{g}$ act on $\mathcal{M}_{\xi}(w)$ by corrspondences.

ii) If $\mathfrak{g}$ is of affine type, and $\operatorname{tr} \xi=0$, then $\mathcal{M}_{\xi}(w)$ is the moduli of $U(n)$-instantons on the ALE space $X_{\xi}$, with monodromy at $\infty$ determined by $w$. (We refer to [KN,N] for all these terms).

In other words, in case (i) Nakajima "cuts down" a Verma module to get an irreducible highest weight module. Unfortunately, (i) and (ii) cannot

\footnotetext{
${ }^{4}$ 'classically' here means after the work of Drinfeld.
} 
occur simultaneously ${ }^{5}$. For example, $\mathcal{M}_{\xi}(0)$ is a point if $\xi$ is generic, but if $\xi$ is generic trace free it is the Hilbert scheme on $X_{\xi}$.

So, if we care about the moduli of instantons on an ALE space, we must do some extra work from [Na]. Obviously, this is the content of this paper, which complements $[\mathrm{Na}]$ even in the case of an ALE space. (Using [Lu2,Na] one can obtain the results above in the quiver language directly. This will appear in [Gr1]).

The point of this series of papers is to use elementary representation theory to obtain information about the moduli space of instantons. In the quiver variety case (for $\xi$ generic), one may reverse this, and use the geometry of quiver varieties to obtain new information about quantum affine algebras.

Specifically, let $\mathfrak{g}$ be a Kac-Moody algebra, and $U_{q} \widehat{\mathfrak{g}}$ the associated quantum affine algebra at central charge 0 [Dr,Gr2]. If $\mathfrak{g}$ is finite dimensional, we can consider the category of finite dimensional representations of $U_{q} \widehat{\mathfrak{g}}$; for the definition for general $\mathfrak{g}$ see $[\mathrm{Gr} 1]$ (these representations have the property that they restrict to a direct sum of integrable highest weight representations of $\left.U_{q} \mathfrak{g} \hookrightarrow U_{q} \widehat{\mathfrak{g}}\right)$. Then, as was discovered by Drinfeld, these representations are not deformations of the analogous representations of $\widehat{\mathfrak{g}}$ (the "evaluation representations" and their tensor products); smaller terms must be added.

The reason for this is that the varieties $\mathcal{M}_{\xi}(w)$ are not zero dimensional; i.e. $H^{*}\left(\mathcal{M}_{\xi}(w)\right)$ is $H^{\Lambda}\left(\mathcal{M}_{\xi}(w)\right)$ plus smaller terms.

Geometrically, we take as our reasonable cohomology theory $K^{G L_{W} \times \mathbf{C}^{*}}\left(\mathcal{M}_{\xi}(w)\right)$, which takes full account of the geometric symmetries of $\mathcal{M}_{\xi}(w)$. Then in [Gr1] it is proved that $K^{G L_{W} \times \mathbf{C}^{*}}\left(\mathcal{M}_{\xi}(w)\right)$, admits an action of $U_{q} \widehat{\mathfrak{g}}$ (see also [Gr2]). This explains the occurance of the middle homology in $[\mathrm{Na}]$.

Also, to continue the advertisement of [Gr1], we construct all of $U_{q} \widehat{\mathfrak{g}}$. Namely, we take equivariant cohomology of the Lagrangian subvariety of $\mathcal{M}_{\xi}(w) \times \mathcal{M}_{\xi}(w)$ consisiting of pairs with the same moment map image. This constructs a piece of $U_{q} \widehat{\mathfrak{g}}$ (and the same variety was independantly discovered by Nakajima in [Na2], where he used it to construct a piece of the enveloping algebra of $\mathfrak{g})$. These pieces fit togethor via the coproductwrite $w=w^{\prime}+w^{\prime \prime}$. This defines a $\mathbf{C}^{*}$ action on $\mathcal{M}_{\xi}(w)$, with fixpoints of the form $\mathcal{M}_{\xi}\left(w^{\prime}\right) \times \mathcal{M}_{\xi}\left(w^{\prime \prime}\right)$. Then one may define a coproduct via localisation to the fixpoints, and this coproduct fits these pieces togethor to produce $U_{q} \widehat{\mathfrak{g}}$.

This coproduct, at the $q=1$ non-affine level, realises the map $L_{w^{\prime}} \otimes$ $L_{w^{\prime \prime}} \rightarrow L_{w^{\prime}+w^{\prime \prime}}$, where $L_{w}$ is the irreducible highest weight module for $\mathfrak{g}$

\footnotetext{
${ }^{5}$ This was explained to me by Greg Moore.
} 
with highest weight $w$.

Finally, we describe the irreducible modules (even at roots of unity) in terms of certain perverse sheaves with nilpotent characteristc variety on Nakajima's moduli space $\mathcal{M}_{0}(w)$.

In the case $\mathfrak{g}=\mathfrak{g l}_{n}$, this construction of the algebra is due to Ginzburg and Vasserot $[\mathrm{GV}]$; the moduli space is due to Beilinson-Lusztig-MacPherson $[\mathrm{BLM}]$; and the coproduct appeared in [Gr3]. That such a geometric picture of the representation theory of $U_{q} \widehat{\mathfrak{g}}$ should exist was conjectured by Drinfeld, on the basis of Kazhdan and Lusztig's description of the representation theory of affine Hecke algebras, which is similar to this. This was explained to me by G. Lusztig, in 1991.

\section{References}

[BLM] A. A. Beilinson, G. Lusztig, and R. MacPherson, A geometric setting for the quantum deformation of $G L_{n}$, Duke Math. J. 62 (1990), 655-677.

[B] R. Borcherds, Vertex algebras, Kac-Moody algebras and the monster, Proc. Natl. Acad. Sci. 83 (1986), 3068-3071.

[Br] J. Briancon, Description de Hilb ${ }^{n} \mathbf{C}\{x, y\}$, Invent. Math 41 (1977), 45-89.

[Dr] V. Drinfeld, A new realisation of Yangians and quantized affine algebras, Soviet Math. Dokl. 36 (1988), 212-216.

[LMNS] A. Losev, G. Moore, N. Nekrasov, and S. Shatashvili, Four-dimensional avatars of two dimensional CFT, talk at USC, March 1995, and paper in preparation.

[FJ] I. Frenkel and N. Jing, Vertex representations of quantum affine algebras, Proc. Natl. Acad. Sci. 85 (1988), 9373-9377.

[FK] I. Frenkel and V. Kac, Basic representation of affine Lie algebras and dula resonance models, Invent. Math 62 (1980), 23-66.

[FLM] I. Frenkel, J. Lepowsky, and A. Meurman, Vertex operator algebras and the monster, vol. 134, Pure and applied math, Academic Press, 1988.

[Fo] J. Fogarty, Algebraic families on an algebraic surface, Am. J. Math 90 (1968), $511-521$.

[Gi] D. Gieseker, On the moduli of vector bundles on an algebraic surface, Ann. of Math 106 (1977), 45-60.

[GL] D. Gieseker and J. Li, Irreducibility of moduli of rank 2 vector bundles on algebraic surfaces, J. Diff. Geom. 40 (1994), 23-104.

[GV] V. Ginzburg and E. Vasserot, Langlands reciprocity for affine quantum groups of type $A_{n}$, Int. Math. Research Notes 3 (1993), 67-85.

[Got] L. Gottsche, The Betti numbers of the Hilbert scheme of points on a smooth projective surface, Math Ann. 286 (1990), 193-207.

[GS] L. Gottsche and W. Soergel, Perverse sheaves and the cohomology of the Hilbert scheme of a smooth algebraic surface, Math Ann. 296 (1993), 235-245.

[Gr1] I. Grojnowski, Representations of quantum affine algebras, Yale University course notes (1994), book in preparation.

[Gr2] _ Affinizing quantum algebras: from D-modules to $K$-theory, preprint 1994, posted to q-alg.

[Gr3] , The coproduct for quantum $G L_{n}$, preprint 1992.

[Gro] A. Grothendieck, Techniques de construction et theorems d'existance en geometrie algebrique IV: Les schemas de Hilbert, Sem. Bourb., Expose 221 (1960). 
[JKS] U. Jannsen, S. Kleiman, and J. P. Serre (eds), Motives, Proc. Symp Pure Math 55 (1994).

[KM] P. Kronheimer and T. Mrowka, Embedded surfaces and the structure of Donaldson polynomial invariants, preprint 1994.

[KN] P. Kronheimer and H. Nakajima, Yang-Mills instantons on ALE gravitational Instantons, Math Ann. 288 (1990), 263-307.

[La] G. Laumon, Un analogue global du cone nilpotent, Duke Math Jour. 57 (1988), 647-671.

[L1] G. Lusztig, Quivers, perverse sheaves and quantized enveloping algebras, J. Amer. Math. Soc. 4 (1991), 365-421.

[L2] _ Affine quivers and canonical bases, Publ. Math IHES 76 (1992), 111163.

[MO] J. Morgan and K. O'Grady, Differential topology of complex surfaces, Springer LNM 1545 (1993).

[Na] H. Nakajima, Instantons on ALE spaces, quivers, and Kac-Moody algebras, Duke Math Jour. 76 (1995), 365-416.

[VW] C. Vafa and E. Witten, A strong coupling test of S-duality, hep-th/9408074 (1994).

Evans Hall, U.C. Berkeley, Berkeley, C A 94720-3840

E-mail address: ian@math.berkeley.edu 